\title{
Group Profile Management in Ubiquitous Healthcare Environment
}

\author{
Maria-Anna Fengou, Georgios Mantas, Member, IEEE, Dimitrios Lymberopoulos, Member, IEEE
}

\begin{abstract}
Nowadays, ubiquitous healthcare is of utmost importance in the patient-centric model. Furthermore, the personalization of ubiquitous healthcare services plays a very important role to make the patient-centric model a reality. The personalization of the ubiquitous healthcare services is based on the profiles of the entities participating in these services. In this paper, we propose a group profile management system in a ubiquitous healthcare environment. The proposed system is responsible for the dynamic creation of a group profile and its management.
\end{abstract}

\section{INTRODUCTION}

Nowadays, the shift from the doctor-centric model toward the patient-centric model has improved the quality of healthcare. In this shift, Ubiquitous Healthcare (UH) plays a major role since the patient-centric model requires the provision of healthcare to anyone, anytime and anywhere without limitations on time and location [1]. In the patientcentric model, the UH services are provided to the patient by a group of entities (medical professionals, relatives, volunteers, etc) who compose dynamically a dedicated service group. The personalization of the UH services can be enabled by the use of the profiles of these entities participating in the $\mathrm{UH}$ environment.

In this paper, we propose a group profile management system in a UH environment considering that each participating entity of the UH environment holds its own profile. We exploit the profiles of the participating entities for the creation and management of a group profile which is responsible to enable the appropriate entities to collaborate for the efficient provision of personalized ubiquitous healthcare services to the patient. The group profile facilitates the dynamic creation of the appropriate group of entities that should be rapidly provisioned and released with minimal management effort. The inception for the creation of a group profile is the patient's health condition. The proposed group profile management system integrates four mechanisms implemented by agents. These mechanisms are responsible for the creation of the group profile and its management.

Following the introduction, this paper is organized as follows. In Section II, related work is presented. In Section III, the definition of a UH environment is given. In section

Maria-Anna Fengou is with Wire Communications Laboratory, Electrical \& Computer Engineering Department, University of Patras, Rio, Patras, GR-26504, Greece (phone:0030-2610996852; e-mail: afengoum@upatras.gr).

Georgios Mantas is with Wire Communications Laboratory, Electrical \& Computer Engineering Department, University of Patras, Rio, Patras, GR26504, Greece (e-mail: gman@upatras.gr).

Dimitrios Lymberopoulos is with Wire Communications Laboratory, Electrical \& Computer Engineering Department, University of Patras, Rio, Patras, GR-26504, Greece (e-mail: dlympero@upatras.gr).
IV, the proposed group profile management is described. In section $\mathrm{V}$, a scenario in $\mathrm{UH}$ environment is deployed. Finally, Section VI concludes the paper.

\section{RELATED WORK}

The work in [2] introduces a new telemedicine framework that addresses the problem of the increased rate of people incoming to the emergency room pointing a health problem. The proposed framework allows medical experts to estimate the real health condition of conditional patients, and then consult them to visit a hospital or not. This framework combines elements such as an expanded electronic medical record enriched with real time acquired context data as well as several actor profiles. This profile's structure allows the telemedicine system to compose multiparty group-working schemes with pre-selected behaviors of all involved actors, devices and services.

The ETSI Recommendations in [3] specifies addressable entities and group. It builds on and extends the Addressable entity and group specified in [4]. Rules, preferences and information data items will sometimes need to refer to entities such as devices, services and people (represented as address book entries). It can be useful for example in rules used for specific situations such as emergency situations. In addition it will also be necessary to refer to groups which may contain any of these other types of entity. Groups are also used for storing and finding contact information in a way that allows them to be more easily found and addressed.

Moreover, in [3] is presented the issues that arise during an emergency situation when many users may wish to share selected information from their profile. For an emergency situation there may be two forms of constraint relating to the sharing of that information. The first issue here is in determining if the current situation is an emergency that would justify the sharing of the emergency-related information. The second issue that needs to be solved here is to determine reliably whether the entity with which the information is being shared is the appropriate person according to his (eHealth) role. When the person or system requiring the information has a "role" specification in his profile that matches the roles specified in the profile user's rules, there is no issue to be solved.

\section{UH ENVIRONMENT}

We consider that a UH environment should be composed, at least, by the following classes of UH entities:

Patient: individual receiving the $\mathrm{UH}$ services, to support independent living and/or using UH services for the care of his or her own health and wellbeing [3]. The state of the patient's health condition is continuously monitored using 
real time collected contextual and bio information, which is gathered by wearable or implanted biosensors as well as by environmental arrays of sensors.

Medical professionals: professionals (e.g. clinicians, doctors, pharmacists) involved in the assessment of patient's health condition and delivery of more specialist care than the provided by carers. They are trained to handle healthcare data and information, to recommend or perform treatments and to use healthcare services and devices [3].

Formal carers: professional providing care for the client. A care professional (e.g. occupational therapist, social worker) is involved in the assessment of patient's health condition delivering of one or more non-medical care roles. The care professional will have a qualification that entitles them to perform various care roles [3].

Informal carers: trainee persons (e.g. relatives, neighbours, friends or volunteers) providing care for the client. This category provides care in a wide variety of situations in a non-professional capacity. They may have a range of competences based on depth to little or no training in the health and care issues of the patients they are involved with. They also may have training in the use of the healthcare services and devices. It is important that the information conveyed from ehealth devices and services is presented in a way that they can understand [3].

In the context of the patient-centric model, patient is the core entity of the UH environment as depicted in Figure 1.

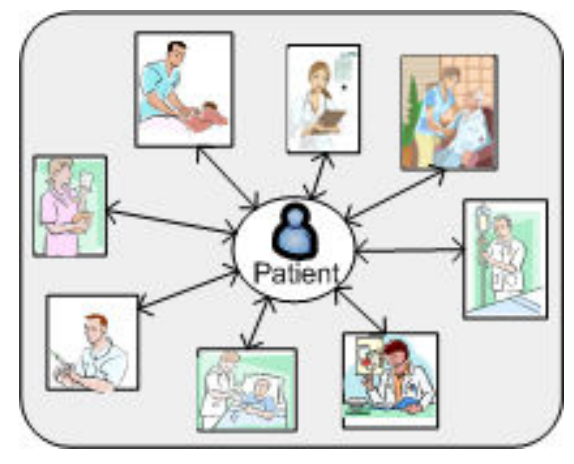

Figure 1. UH Environment

\section{Group Profile Management}

Each of the above participating entities in a UH environment has a profile. The profile may include information such as demographic data, preferences, contextual information, etc. It is not only important that the profile contains all the relevant information, but also that it is accurate and up-to-date.

The state of the patient's health condition is the inception for a UH service to be delivered. The health condition is described at a high-level. It is not intended to give detailed information about the current health status of the person, but the health status to be used in the creation of rules or for informing other people such as relatives and friends. According to [3], the health condition can be characterized by four discrete states:
- $\quad$ well: the patient feels well;

- mild-condition: a condition that does not affect strongly the patient;

- stronger-condition: a condition that strongly affects the patient;

- emergency: in emergency.

These states are depicted in Figure 2.

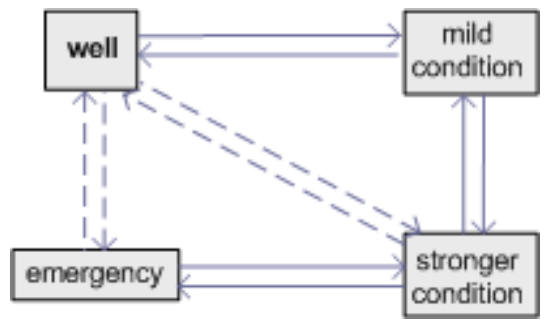

Figure 2. The four potential states of patient's health condition and the respective transitions from one state to another

At a time, the patient's healthcare condition can be in only one state. At each state, certain UH services are provided to the patient by certain entities. For example, in case the state of the patient's health condition is transitioned to the "mild condition" (e.g. experiencing significant changes in sensory ability), it may be necessary to alert a social worker or a relative even if the patient has not detected that his health condition has reached this state in order to ensure that services being used are adjusted accordingly. Alternatively, when the wearable sensors detect that the patient's physiological measures have exceeded the threshold values, a doctor should be alerted or an emergency service as the patient's health condition has reached the "emergency" state.

For the provision of such services, the determination of the entities that will be activated to form dynamically the appropriate group for each situation is of great importance. We consider that the patient's health condition functions as the convener of a session and the participating entities as the participants of a session respectively [5]. Each role has its competences as when a conference is convened.

\section{A. Group Profile Structure}

Each state of the patient's health condition corresponds to a group profile. The group profile integrates two main types of information; the roles and the rules. The roles correspond to the participating entities that are essential for each group in order to accomplish its overall tasks. The group consists of entities with discrete roles in the provision of the UH service. The rules are statements required for the selection of the appropriate entities. The selection is based on the roles' requirements as well as the preferences of the patient. In Figure 3, it is presented the mapping of the roles for each state of the health condition.

There are depicted $K$ Roles and $L$ States. The assigned numbers depend on the personalized service. We consider that there are $\mathrm{L}=4$ states in our approach following the defined states in [3]. 


\begin{tabular}{|c|c|c|c|c|}
\hline & State 1 & State 2 & $\ldots$ & State L \\
\hline Role 1 & & ptr) & & \\
\hline Role 2 & 7 & ptr & & \\
\hline & & & (ptr) & \\
\hline Role K & & & & \\
\hline e.g. & e.g. & 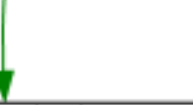 & & e.g. \\
\hline \begin{tabular}{|c|} 
Catalog \\
Doctor \\
Doctor \\
\end{tabular} & & $\begin{array}{l}\text { Catalogue } \\
\text { Social worker 1 } \\
\text { Social worker } 2 \\
\end{array}$ & & $\begin{array}{l}\text { Catalogue } \\
\text { Relative I } \\
\text { Relative 2 } \\
\end{array}$ \\
\hline \begin{tabular}{|l} 
Doctor \\
\end{tabular} & & Social worker n & & Relative n \\
\hline
\end{tabular}

Figure 3. Mapping of the roles for each state of the health condition

For each state of the health condition, we consider that there are $K$ roles that need to participate in order to form the group and provide support to the patient performing specific activities (e.g. apply treatment guidelines). Each role corresponds to one of the defined participating entities. In each role, there is a pointer referencing to a catalogue stored in the patient's profile. This catalogue contains a list of potential entities that are defined as the patient's preferred healthcare group that treats him and consult him.

The selection of a potential entity from the catalogue is based on a set of rules that are related both to the preferences of the patient and to the availability of the participating entity. These rules are stored in the profiles of the patient and the participating entities respectively. For example, a rule stored in the patient's profile could be for specifying the priority of a potential entity from the most preferred to the least preferred.

For each role, there are also general policies that define what access the entity may have to the patient's profile. These policies can be overwritten if the patient has defined related sharing preferences stored in his profile. The patient can define three different types of access: read, write and delete.

- The read access is used to express his wishes for how a profile data can be shared regarding the read access [3].

- The write access is used to express his wishes for how a profile data should be shared regarding the write access [3].

- The delete access is used to express his wishes for how a profile data should be shared regarding the delete access [3].

In these three types of access, profile data a) can be shared without needed confirmation b) can be accessed after the patient has confirmed that the specified addressable entity has the rights c) cannot be accessed by other than rules within the profile system [3].

\section{B. The Proposed Group Profile Management System}

The proposed Group Profile Management System integrates the following four mechanisms: the Event Handler, the Role
Assignment, the Group Profile Creation and the Group Profile Update. These mechanisms are implemented by agents and are depicted in Figure 4.

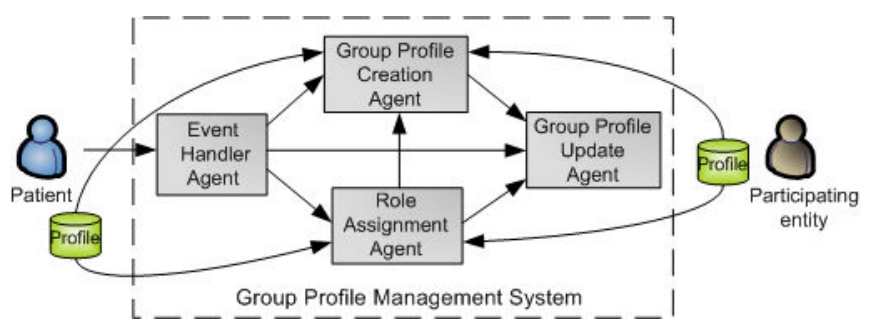

Figure 4. Group Profile Management System

\section{Event Handler:}

This mechanism is responsible to detect and handle the events that trigger the creation of the group profile. These events are related to the transition of the patient's health condition from one state to another. An event can be defined as a significant change in specific bio or contextual information captured by the corresponding bio or contextual sensors. This mechanism is used to evaluate the events in order the appropriate decisions to be taken. For example, an event can denote that the patient needs a medical advice or attention and care due to aggravation of his health condition. Thus, this mechanism activates the creation of the group profile.

\section{Role Assignment:}

This mechanism assigns roles to the potential entities of the group in order the creation of the group profile to be achieved. It defines the roles that are required and invites the potential entities in order to build the group. Each role has its competences as when a conference is convened. The patient (his health condition) and the formed group function as the convener and the participants of the session respectively.

\section{Group Profile Creation:}

This mechanism is used for the creation of the group profile. The activation of this mechanism is event-driven. Whenever an event (e.g. the patient's health condition is deteriorated) is detected by the event-handler, the group profile is created based on the pointers that are stored in the patient's profile. The entities of the group that will participate are specific. The information stored in their profiles determines whether an entity meets the conditions in order to participate. A coordinator is also defined in the group depending on the roles that participate for the provision of a UH service. For instance, if an emergency service is delivered, then a medical professional (e.g. doctor) will be responsible to coordinate the group in order all the participating entities to collaborate efficiently.

\section{Group Profile Update:}

This mechanism is used for the update of the group profile. After the activation of the group profile, collaborative actions may follow such as decision-support and profile-sharing depending on the patient's health condition. In addition, the attributes of each participant may change dynamically over time. The participants' presence is constantly verified. Moreover, if a new event is detected by 
the event handler, an additional role may be required and as a result another entity will be added in the already formed group.

\section{DESCRIPTION OF SCENARIO}

We consider the case of a Smart Home equipped with the appropriate sensors for gathering context and bio information as well as integrates the essential residential networks for supporting a UH system for providing monitoring services to a cardiac patient remotely. The patient is being monitored by the system in order any critical event to be detected timely.

Based on this scenario, we consider the use of the patient's profile, a number of profiles of doctors and nurses related to the patient and a set of profiles of the patient's relatives. Moreover, the proposed Group Profile Management System is used integrating the following mechanisms.

Initially, the "Event Handler" mechanism is used to detect any event that denotes a change in the state of the patient's health condition. Afterwards, when such an event is detected, the "Role Assignment" mechanism is used to define the participating entities of the group profile.

Furthermore, the "Group Profile Creation" mechanism, is responsible the patient's profile to be accessed for acquiring the identities of the entities that are related to the patient and will participate in the group that supports the UH service. Firstly, a request (Dreq) is sent to the patient's profile whose response (Dres) returns the doctors who are engaged with this patient and are already subscribed to this service. Then, queries are sent to these doctors' profiles in order to detect their availability and to bind one of them. Similarly, all the participants (e.g. relative, nurse) of the healthcare service are bound. As a result, a group comprising a doctor, a nurse and a relative, is formed. Figure 5 depicts an example of a creation of the group profile.

If the current state of the patient's health condition justifies the sharing of the emergency-related information from the patient's profile, this information may be necessary to be shared with a participating entity. Where the participating entity requiring the information has a "role" specification in the patient's profile that matches the roles specified in the profile patient's rules, there should be no problem. The sharing will be based both on general policies that define what access the participating entity may have to the patient's profile and on the patient's haring preferences.

Finally, the "Group Profile Update" mechanism can be implemented in an exceptional case either when a participating entity (e.g. the nurse) has to be replaced by another nurse or when the state of the cardiac's health condition changes requiring an additional entity to participate the group.

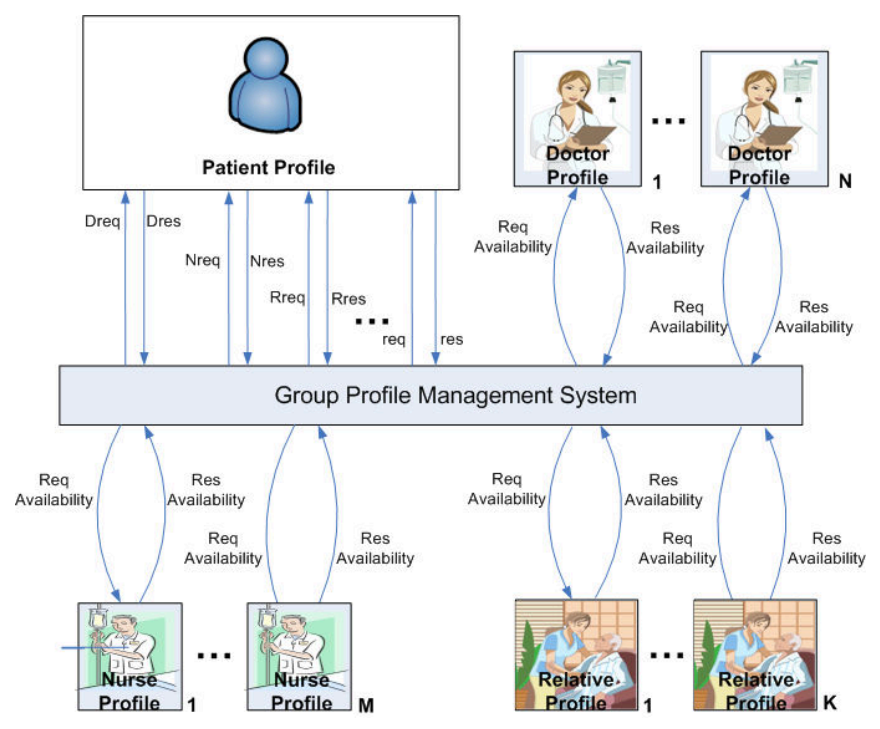

Figure 5. The creation of a group profile

\section{CONCLUSION}

In this paper, we have proposed a group profile management system for the provision of personalized UH services. The proposed system integrates four mechanisms, the Event Handler, the Role Assignment, the Group Profile Creation and the Group Profile Update, which are responsible for the creation and management of group profiles. The proposed group profile management system exploits the profiles of all participating entities. Finally, as future work, we plan to implement the proposed group profile management system using agent technology and evaluate its performance regarding the quality of the provided personalized UH services in case that a wide range of participating entities are involved.

\section{REFERENCES}

[1] U. Varshney, "Pervasive Healthcare: Applications, Challenges And Wireless Solutions," Communications of the Association for Information Systems, AIS Electronic Library, vol. 16, no. 1, article 3, pp. 57-72, 2005.

[2] M.-A. Fengou, T. Panagiotakopoulos, S. Fengos, N. Lazarou, D. Lymberopoulos, "A New Telemedicine Framework Handling the Emergency Room Overload," in Proc. 10th IEEE International Conference on Information Technology and Applications in Biomedicine, Corfu, 2010.

[3] ETSI ES 202642 V1.1.1, "Human Factors (HF); Personalization of eHealth systems by using eHealth user profiles (eHealth)," 2010.

[4] ETSI ES 202746 V1.1.1, "Human Factors (HF); Personalization and User Profile Management; User Profile Preferences and Information," 2010.

[5] E. Karavatselou, G.-P.Oikonomou, C. Chassomeris, V. DaneliMylonas, and D. Lymperopoulos, "OTE-TS-A New Value-Added Telematics Service for Telemedicine Applications," IEEE Transactions on Information Technology in Biomedicine, Vol.5, No.3, September 2001. 